\title{
STM Image Artifacts on Highly Ordered Pyrolitic Graphite That Could Be Mistaken for Carbon Nanotubes
}

\author{
Morewell Gasseller \\ Xavier University of Louisiana, New Orleans, USA \\ Jessica Ritchie \\ Mercyhurst University, Erie, USA \\ Erin McCarthy \\ Pennsylvania State University, University Park, USA
}

\begin{abstract}
Since the introduction of the national nanoscience initiative in 2000, many high schools across the country have started to introduce nanotechnology in their curriculum. The benchtop easy scan scanning tunneling microscope (STM) system is one of the most common instruments used in this nanotechnology classroom. This paper highlights some of the challenges involved in interpreting STM data when graphite is used as the substrate. Highly oriented pyrolytic graphite (HOPG) is a common substrate for STM studies of carbon nanotubes. It is an ideal surface for STM, because it is easily cleavable by adhesive tape, resulting in large, atomically flat planes that are relatively inert and electrically conducting. Despite these attractive attributes, the cleavage of HOPG surfaces also generates a variety of artifacts, some of which are elongated structures similar to the carbon nanotubes being investigated. Some even exhibit periodicities that mimic the atomic structures expected in the carbon nanotubes. In our investigation of carbon nanotubes deposited on a graphite substrate, we observed and catalogued many of these commonly known filament-like artifacts.
\end{abstract}

Keywords: nanotechnology, carbon nanotubes, scanning tunneling microscopy, graphite

\section{Introduction}

The scanning tunneling microscope (STM) is a powerful probe with high spatial resolution for studying the surface topography and electronic properties of conducting samples. In the past decade, STM has drawn considerable interest from researchers and educators wishing to study carbon nanotubes. The increased use of STM to investigate the electronic and structural features of carbon nanotubes on a graphite substrate prompted us to investigate the presence of filament-like artifacts on the surface of cleaved graphite. To do this, we used STM to image single walled carbon nanotubes (SWCNT) deposited on a graphite substrate. Over the years, we have observed and catalogued many of these filament-like artifacts. The data presented here show simple ways of differentiating the actual carbon nanotube from these filament-like graphite artifacts in STM experiments.

Highly oriented pyrolytic graphite (HOPG) is a common substrate for STM studies of carbon nanotubes (Riveraa et al., 1995; Venema et al., 1998; Koós et al., 2003; Biró et al., 1997). It is an ideal surface for STM,

Morewell Gasseller, assistant professor, Department of Physics, Xavier University of Louisiana.

Jessica Ritchie, undergraduate student, Department of Physics, Mercyhurst University.

Erin McCarthy, graduate student, Department of Biochemistry, Pennsylvania State University. 
because it is easily cleavable by adhesive tape, resulting in large, atomically flat planes. Whereas most surfaces will contaminate very quickly in air to form a non-conducting surface layer that will prevent tunneling, graphite is very stable and will not oxidize due to the planar nature of the carbon-carbon bonds. When a graphite sample is cleaved, there are no highly reactive dangling bonds at the surface. Typical planes range in size from hundreds of nanometers to tens of microns. Despite these attractive attributes, the cleavage of HOPG surfaces also generates a variety of artifacts, some of which are elongated structures similar to the carbon nanotubes being investigated. Some even exhibit periodicities that mimic the atomic structures expected in carbon nanotubes (Chang \& Bard, 1991).

\section{Experimental Procedure}

The STM used was made by Nanosurf Instruments Inc.. SWCNT powder was purchased from Nanostructured and Amorphous Material Inc.. The nanotube samples were prepared for STM imaging by sonicating the carbon powder in methanol and placing a few drops on a HOPG substrate. The STM imaging and I-V spectroscopy were performed in air using an electrochemically etched tungsten tip. Typical tip-sample voltages and tunneling currents used for imaging were $50 \mathrm{mV}$ and $1.0 \mathrm{nA}$, respectively. We scanned hundreds of graphite surfaces, some which were just bare HOPG and others on which SWCNTs have been deposited. A result of this large number of images was a collection of some common and rare HOPG artifacts.

\section{Common Artifacts}

Several STM observations of filament-like artifacts on graphite have been catalogued in the literature (Chang \& Bard, 1991; Sinitsyna \& Yaminsky, 2006; Simonis, Goffaux, Thiry, Biro, Lambin, \& Meunier, 2002; Gan, Chu, \& Qiao, 2003). We encountered many of these during our STM studies of graphite surfaces that were bare and surfaces on which carbon nanotubes were deposited. Here, we discuss a few of these. First, the rather simple and abundant step edge (Sinitsyna \& Yaminsky, 2006) formations found in virtually every STM scan can occasionally appear to be more than just the edges of carbon sheets. Single atomic step edges are rather easy to identify because of their small height of just a few angstroms, like one shown in Figure 1(a) and its cross section shown in Figure 1(b). Edges of several graphite sheets may be as high as a few nanometers. These stacked sheets will often give the illusion of structure when the break is not straight. Especially with image processing artifacts, such as line by line subtraction, the edge may appear to have the structure and depth of a carbon nanotube. Figure 1(c) shows an example of such an artifact. The step height in Figure 1(d) is about $0.8 \mathrm{~nm}$ and graphite's interlayer spacing is about $3.3^{\circ} \mathrm{A}$, indicating that this step is a height of about two or three stacked layers. The rounded and jagged breaks on the edge mimic the edge of an elongated object like a carbon nanotube adsorbed on the surface. Line by line subtraction, an image post-processing function that is often performed by the software during data acquisition, gives the illusion of depth.

Other common artifacts are grain boundaries (Simonis et al., 2002; Gan et al., 2003). These occur at interfaces of graphite grains. Most grain boundaries are not imaged without atomic resolution. Figure 2(a) shows what we believe to be a grain boundary artifact. Grain boundaries may exhibit structure that has a periodicity mimicking a carbon nanotube atomic structure. The surface is flat, but the electronic structure leads to an apparent height that is finite in the STM imaging. This phenomenon is described in reference (Simonis et al., 2002). The feedback loop of the STM in constant current mode will pull the tip away from the surface when it detects current exceeding the set point. Because the imaging software plots this as a topographical height, the 
electronic structure leads to an imaging artifact that looks like an adsorbed nanotube. As shown in Figure 2(b), these artifacts have an apparent height of just a few angstroms, in contrast to carbon nanotubes apparent heights of a few nanometers.
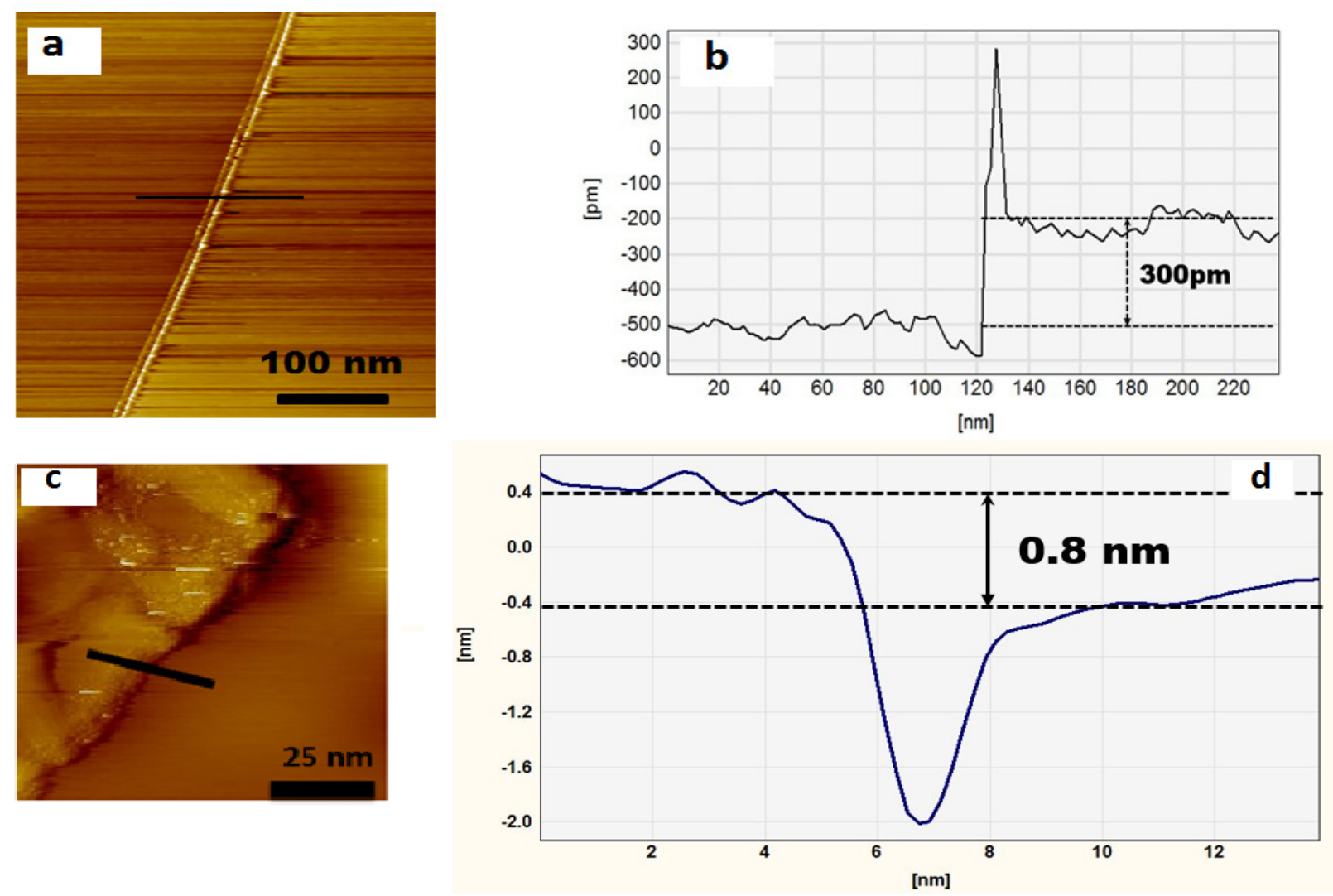

Figure 1. (a) STM topography image of a single atom step edge on a HOPG substrate. As evident in the profile in (b), the edge is about $300 \mathrm{pm}$ in height, which is equivalent to about one atomic layer. (c) STM topography of a rough step edge. As evident in the profile in (d), the edge is several layers in height. To produce the plots in (b) and (d), a best fit line in the direction of the cut was subtracted to correct for the background slope. This subtraction makes the step edge easier to identify. The images were recorded in the constant-current mode with a bias voltage of $50 \mathrm{mV}$ and a tunneling current of $1.0 \mathrm{nA}$.
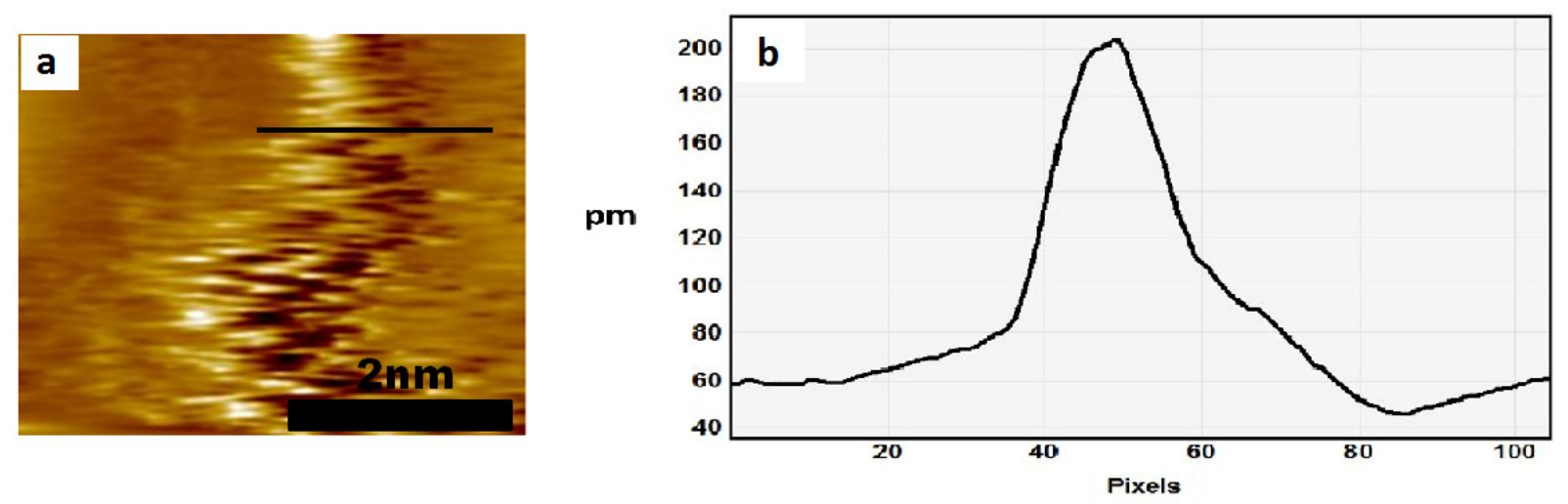

Figure 2. (a) Representative STM topography image of grain boundaries encountered during our studies. (b) A cross section of the artifact along the black line shown in (a). The STM image shown in (a) were recorded in the constant-current mode with a bias voltage of $50 \mathrm{mV}$ and a tunneling current of $1.0 \mathrm{nA}$. 


\section{Artifacts Created by the Scan Head}

Another artifact we observed is as shown in Figure 3. It is an elongated structure that can be mistaken for a carbon nanotube. However, it cannot be a carbon nanotube, because it is very narrow, only about 3.5 angstroms in diameter whereas the smallest nanotube is about 10 angstroms. Because this artifact has not been reported anywhere in literature, we believe that it is an artifact coming from the microscope scan head. This artifact is most likely related to the zero crossing of the analogue to digital converter (ADC) (Bai, 1992). When crossing zero, the ADCs have to work hard: The smallest positive value is 00000001 (in case of an 8 bit binary data). The highest negative value (closest to zero) is 1111 1111. This means that most bins have to switch between 0 and 1 when the scan position goes from negative to positive. This defect can be circumvented by measuring at an offset so the image does not go through the origin of the scanner range. Slope adjustment can also help to get rid of this artifact.
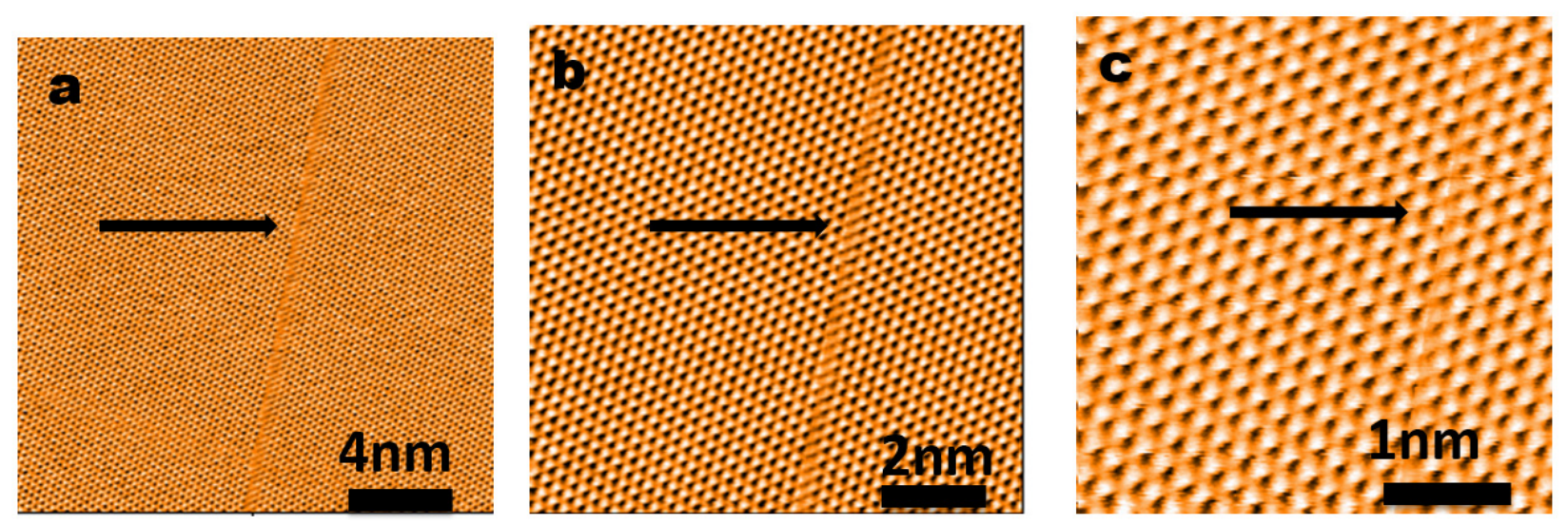

Figure 3. Artifacts caused by the scan head. (a) STM atomic resolution topography image of HOPG. The image was taken at a scan range of $16 \times 16 \mathrm{~nm}$. The black arrow shows the position of the artifact. (b) and (c) show the same artifact when the scan range was reduced to $8.0 \times 8.0 \mathrm{~nm}$ and $4.0 \times 4.0 \mathrm{~nm}$, respectively. This artifact is most likely related to the zero crossing of the ADC converter. The STM images were recorded in the constant-current mode with a bias voltage of $50 \mathrm{mV}$ and a tunneling current of $1.0 \mathrm{nA}$.

\section{The Zipper Artifact}

We also identified a long structure resembling a zipper on a hopg surface on which carbon nanotubes were deposited. This artifact is shown in Figure 4(a). The artifact is a few microns long and had a periodicity of about $10.0 \mathrm{~nm}$ when measured in a cross section along the axial direction. The symmetric periodicity and dimensions are inconsistent with any known carbon nanotube properties. Furthermore, the width of the zipper structure $(20-30 \mathrm{~nm})$ is much larger than the diameter of the carbon nanotubes we deposited on the hopg (1-5 $\mathrm{nm})$. The zipper structure shown here has a morphology resembling that reported for grain boundaries (Červenka \& Flipse, 2009; Biró, Thiry, Lambin, Journet, Bernier, \& Lucas, 1998; Clemmer \& Beebe, 1991). However, it has more substructure than any artifact previously reported in the literature (Heckl \& Binnig, 1992). In those reports, the authors showed that torn sheets of graphite rotated at specific angles relative to one another, which resulted in the formation of an interface that is imaged by STM as a filament-like periodic grain boundary. We only observed this artifact on one HOPG sample on which carbon nanotubes were deposited. Thus, we could not rule out the possibility that the deposition method contributed to the formation of this artifact. 

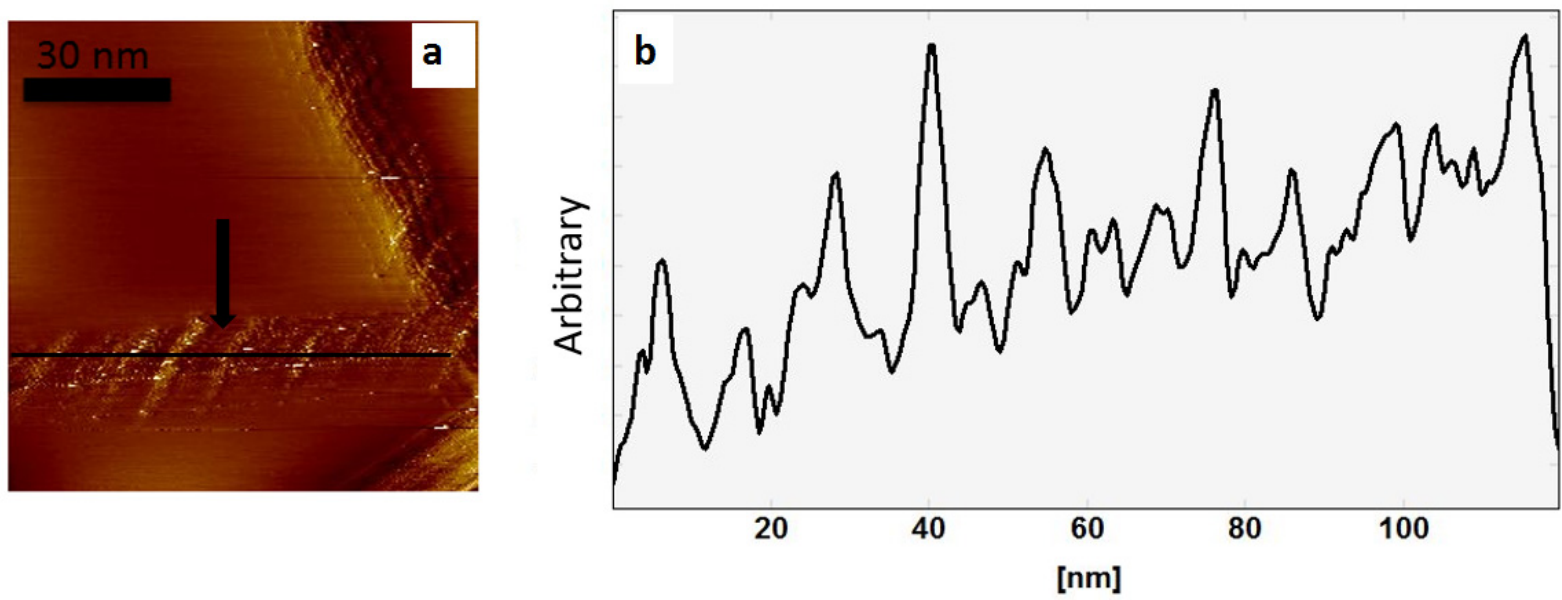

Figure 4. (a) STM topography image of a zipper structure. The artifact is a few microns long and had a periodicity of about $10.0 \mathrm{~nm}$ when measured in a cross section along the axial direction as shown in (b). The symmetric periodicity and dimensions are inconsistent with any known carbon nanotube properties. The width of the zipper structure (20-30 $\mathrm{nm}$ ) is much larger than the diameter of the carbon nanotubes we deposited on the HOPG substrate. The images were recorded in the constant-current mode with a bias voltage of $50 \mathrm{mV}$ and a tunneling current of $1.0 \mathrm{nA}$.

\section{Carbon Nanotubes}

We also observed images that are consistent with the carbon nanotubes we are investigating. The STM images shown in Figure 5 have been filtered to cut low frequency components in order to increase the contrast in the image. The size of the elongated structures shown here are consistent with the size of the nanotubes deposited on the HOPG samples. This is confirmed by taking cross-sections of these topography images. These images were only obtained from those samples on which SWCNT have been deposited. In Figure 5(c), the graphitic sidewall pattern on the carbon nanotubes is atomically resolved.
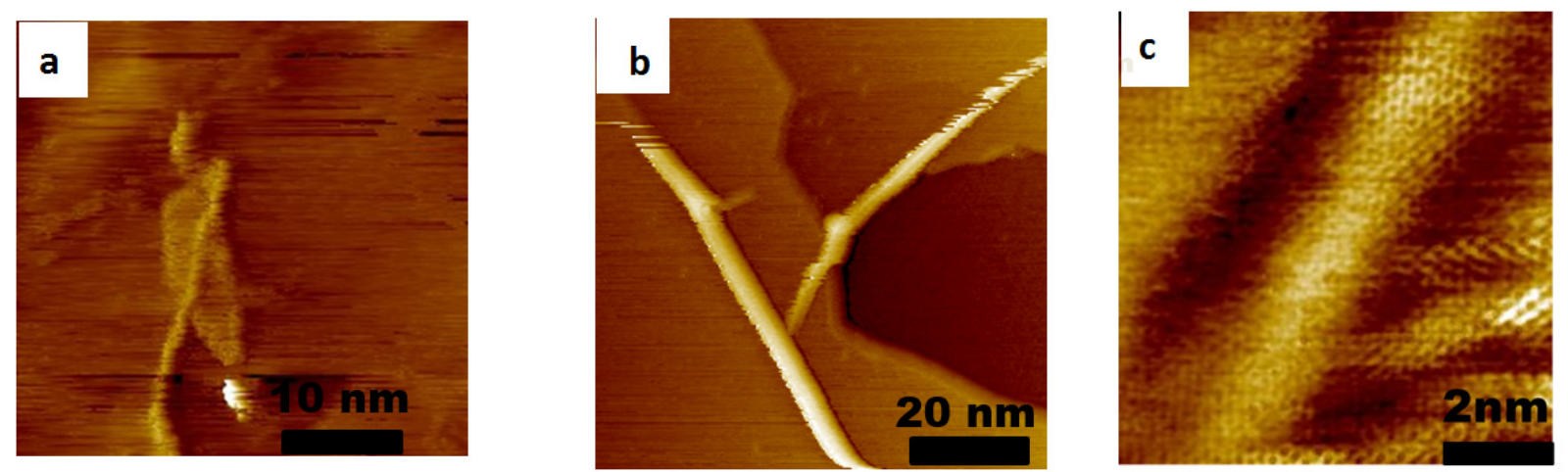

Figure 5. (a) and (b) show STM images of isolated SWCNT on a HOPG substrate (c) shows atomically resolved STM images of SWCNTs. The images were recorded in the constant-current mode with a bias voltage of $50 \mathrm{mV}$ and a tunneling current of $1.0 \mathrm{nA}$.

\section{Conclusions}

These results demonstrate that the surface of cleaved HOPG contains artifacts that resemble carbon nanotubes or other elongated objects when imaged with STM. The presence of these artifacts demands a careful analysis of the images in order to differentiate the actual nanotube image from an artifact whenever HOPG is 
used as a substrate. The combination of atomically resolved images and STM spectroscopy opens the possibility to experimentally investigate the relation between the chiral structure of a nanotube and its electronic properties.

\section{References}

Bai, C. (1992). Scanning tunneling microscopy and its applications (2nd ed., pp. 98-99). Shanghai: Springer Shanghai Scientific and Technical Publishers.

Biró, L. P., Lazarescu, S., Lambin, P., Thiry, P. A., Fonseca, A., Nagy, J. B., \& Lucas, A. A. (1997, November). Scanning tunneling microscope investigation of carbon nanotubes produced by catalytic decomposition of acetylene. Physical Review $B, 56,12490$.

Biró, L. P., Thiry, P. A., Lambin, P., Journet, C., Bernier, P., \& Lucas, A. A. (1998, December). Influence of tunneling voltage on the imaging of carbon nanotube rafts by scanning tunneling microscopy. Applied Physics Letters, 73, 3680-3682.

Červenka, J., \& Flipse, C. F. J. (2009). Structural and electronic properties of grain boundaries in graphite: Planes of periodically distributed point defects. Physical Review B, 79, 195429.

Chang, H., \& Bard, A. J. (1991, June). Observation and characterization by scanning tunneling microscopy of structures generated by cleaving highly oriented pyrolytic graphite. Langmuir, 7, 1143-1153.

Clemmer, C. R., \& Beebe, T. P. (1991, February). Graphite: A mimic for DNA and other biomolecules in scanning tunneling microscope studies. Science, 251, 640-642.

Gan, Y., Chu, W., \& Qiao, L. (2003, August). STM investigation on interaction between superstructure and grain boundary in graphite. Surface Science, 539, 120-128.

Heckl, W. M., \& Binnig, G. (1992, July). Domain walls on graphite mimic DNA. Ultramicroscopy, 42-44, 1073-1078.

Koós, A. A., Ehlichb, R., Horváth, Z. E., Osváth, Z., Gyulaia, J., Nagy, J. B., \& Biró, L. P. (2003). STM and AFM investigation of coiled carbon nanotubes produced by laser evaporation of fullerene. Material Science and Engineering C, 275-278.

Riveraa, W., Perez, J. M., Ruoff, R. S., Lorents, D. C., Malhotra, R., Lim, S., ... Pinizzotto, R. F. (1995, March/April). Scanning tunneling microscopy current-voltage characteristics of carbon nanotubes. J. Vac. Sci. Technol. B, 13(2).

Simonis, P., Goffaux, C., Thiry, P. A., Biro, L. P., Lambin, P., \& Meunier, V. (2002). STM study of a grain boundary in graphite. Surface Science, 511, 319-322.

Sinitsyna, O. V., \& Yaminsky, I. V. (2006). Atomic resolution probe microscopy of the graphite surface. Russ. Chem. Rev., 75(1), 23.

Venema, L. C., Wildöer, J. W. G., Dekker1, C., Rinzler, G. A., \& Smalley, R. E. (1998). STM atomic resolution images of single-wall carbon nanotubes. Physical Review A, 66, 153-155. 